\title{
A brief analysis on the new trend of logo design in the digital information era
}

\author{
Wenyan Zhao ${ }^{1, a}$ \\ ${ }^{1}$ School of Art and Design, Jingdezhen Ceramic Institute, Jingdezhen, China \\ avichy119@163.com
}

Keywords: Digital information era, Logo design, Diversification, Development trends

\begin{abstract}
The digital expression form and the new media technology application make the brand logo tend to be diversified in art style and expression form and also show a new development trend under the dissemination of the current social ideology and emerging media. The reform in the digital information era also promotes the continuous changes of the logo design trend. This paper aims to look forward to the development future of logo design through the research and analysis of the personalized, flattening, colorful and diversified design trend of logo design in the digital information era.
\end{abstract}

\section{The design background in the digital information era}

With the development of science and technology, digital information technology has rapidly swept the world. The strong information wave promotes great changes of the current society and profoundly changes various fields, including politics, economy, culture, education, literature and art. The digital information era is different from any other era. The technology pace in the digital information era is faster and faster. From PC to the Internet to the big data era of the mobile Internet, the fast development of new technology and new media profoundly influences and changes various aspects in our work and life. In the media, the Internet and mobile Internet make information transmission faster and more diversified, so as to shorten the difference between the traditional media and the new media. And the transmitters and audience tend to be interactive and diversified. The rapid development of digital media technology brings great revolution to the design field. And the digital expression form and new media technology application make designers be able to use richer computer language to express design works. The development of various technology and media shows a wider development space of logo design for us.

\section{Outline of logo design}

The logo design, referred to as LOGO design, is a kind of symbolic visual communication symbol, representing specific objects and symbolic context. Logo design is the art in a little space. It shall create a complete and beautiful graph in a limited plane and meanwhile it shall be easy to identify and unique. In addition, the logo shall accurately transmit the internal information content of the enterprise or brand. Therefore, as the foundation and basis of brand visual identification system, the logo is an important medium to transmit brand information. The brand logo reflects the brand image and determines the direction and trend of the overall development of visual identification system. The brand logo expresses the spiritual idea and cultural connotation of the brand through concise and simple visual image and skillfully uses symbol recognition, association and thinking ability to convey unique brand information to the audience. The logo is the product of economic development in a certain stage. It has deep times imprint. Compared to word language, the logo can fastly and directly express brand personality and convey brand information with its visual and graphical characteristics to enhance the brand memory of consumers. In order to accurately use visual image to convey the brand value and brand characteristics, the design style of logo design continues to evolve with the aesthetics of each era. 


\section{The trend of logo design in the digital information era}

In the digital information era, the attention economy prevails. The market competition is more intense, the social trend is rapidly changing and the consumers' psychological needs and aesthetic tastes are also constantly changing. The audience are no longer satisfied with the traditional consumption mode and consumption channels. The changes of consumer attitudes and the improvement of aesthetic ability undoubtedly put forward higher requirements on the brand building. If a brand refuses to make progress, it will be eliminated by the market. Thus, in order to get consumer recognition, various companies constantly update their brand images to increase the competitiveness of their brand images.

With the improvement and innovation of printing technology, the digital expression form and the new media technology application make the logo design break through the bottleneck of the old technology and inspire more possibilities in design form. In the digital information era, the contemporary designers continuously change the traditional design ideas and methods and explores new design grammar in the new era. Under the dissemination of the current social ideology and emerging media, logo design gradually tends to be diversified in art style and expression form and also shows a new development trend.

\subsection{The form of logo design develops to personalization from generality.}

In the history of logo design, the modernism design style with "Bauhaus design" as the representative has had a profound impact on logo design for a long period of time. The form of logo design mainly is simple and abstract geometric figure and it emphasizes high rationality and unification in model. For the style, the basic form of design combination is circular, square and triangle and the model is highly abstract with a strong sense of form. Some well-known brand logos in 20th century were regarded as classic cases of logo design with their simple and rational design style. Many enterprises followed and imitated these cases. In the course of time, with the economic prosperity and increasing similar enterprises, the model combination of the logo design geometric shape is easy to be repeated or similar and the logo is increasingly convergent. It can't highlight the distinctive brand personality, but it will lead to the ambiguity in brand identification and obstacles in visual communication.

In the digital information era with visual image as the center, the visual communication has increasingly become the main information transmission means. Under the social environment with the serious shortage of attention resources, the audience's emotional needs are increasingly concerned by the brand. The people-oriented emotional design principle makes the design style of brand logo pursue personalization. For example, the banking industry regards the logo design of Bank of China as the industry benchmark. The logo design of many banks uses the copper coin model with circular exterior and square interior to highlight the industry attributes and the model is identical. And the Bank of Hangzhou is unique in industry logo. The logo model abandons the traditional coin elements and uses the diamond shape to express the wealth cohesion and sense of worth. Supplemented by the silhouette of flowers and bird as the decoration, it expresses the charm of "paradise on earth", Hangzhou. The Bank of Hangzhou breaks the homogeneous design of industry logo. With humanistic aesthetic emotion, natural and distinctive personality expression, the brand is more likely to be favored by consumers.

\subsection{The logo design style develops to flattening from three-dimensional style.}

The development of logo design is always circular between the plane and stereo. With the rapid development of information technology, the computer software technology constantly updates and improves. The logo design form has converted to multi-dimensional stereoscopic space form from two-dimensional logo model. The main function of early logo is to emphasize the recognition and guidance. The form mainly is simple planar design. But with the development of technology and popularity of HD screen, the logo begins to develop to richer three-dimensional form from the plane. People pay more attention to the rich visual feeling of logo and use the strong function logo shape of design software to convert into gradient, bleaching and rich artistic effects in various levels, so as 
to increase the form beauty of logo design.

But the complexity of logo model also affects the rapid identification and weakens the function. The current Internet society reflects the fast pace of life. It is concise and lively, and can fastly recognize the logo design style. So the flattening logo rises in response to proper time. The flattening design gives up complicated decorative effects, such as shadow, penetration, texture, gradual change, etc. The boundaries of all elements are smooth and clean without any eclosion or shadow. The graph shall be both concise and artistic; the color shall be pure, harmonious and as well as striking. The flattening design style expresses the logo through abstract, simplified and symbolized design elements and it puts forward higher requirements on the recognition and artistry of logo design. For example, the new logo released by Pizza hut reflects the flattening design trend. The logo converts the expression form of classic classic form into the flattening style with seal effect from shiny three-dimensional effect and combines the pizza shape with the straw hat graph to highlight the classics and purity expressed by brand spirit.

\subsection{The color of logo design develops to various from single color.}

The logo color in the traditional logo design is mainly three-primary colors. The gradient and bleaching processing can't be used and the use of color is as simple as possible. The logo design uses CMYK color mode, so that the printed copy can improve the color reduction degree. Therefore, limited by carrier communication and technical means, the color expression of logo design has certain limitations.

In recent years, with the rapid development of science and technology, digital media has quickly entered people's lives. The logo as the expression logo has undergone fundamental changes. Due to the further development of digital printing technology, the color application of logo also breaks through the original limitations. The current digital media uses the RGB color model. The range that can be expressed by RGB color model is more extensive than CMYK color mode. The color use in logo design is not limited in three-primary colors. It can use more gradient colors and richer texture. In the past, we can only rely on traditional graphic and paper media. But now, everyone has at least one mobile terminal and the electronic screen has become a new carrier of print media. The rapid development of the Internet also accelerates the process. Meanwhile, the carrier change also provides greater space for the logo manifestation. The use of diversified colors increases the brand image fashion, which is more in accordance with the aesthetic needs of contemporary audience.

\subsection{The logo design function develops to diversified development from simple development.}

The digital information era is a digital information storage, processing and exchange era. The changes brought by the digital information era for our lives are comprehensive from the life style to conveyed ideas and from thinking way to design concept. The digital age creates a new art form. The visual communication in the digital information era is no longer confined to the visual information brought by paper printing. The ideology of film, photography, advertising, games, multimedia, Internet and other media also put forward higher requirements on the functional diversification of logo design.

With the expanding brand scale, the brand logo design covers increasingly more fields and the combination forms are more complex. It puts forward higher requirements on the function of logo design. Through the setting of the logo form and color, combination mode and auxiliary graph, the logo design uses integrated concept and diversified combination to unify the enterprise internal structure system and style. While maintaining the system integrity and consistency, it also keeps the individuality and the characteristics of each subsystem, enhances the flexibility and diversity of brand visual image and skillfully forms diversified brand visual identification system.

\section{Conclusion}

The ever-changing digital information era continuously affects the development trend of logo design. The digital expression form and new media technology application make the modern logo design break through the barriers of old technical limitations. The inherent design method and 
symbolic concept are gradually broken and the design methods that are always used in logo are also deconstructed. Compared to the traditional logo design, the modern logo design integrates more personalized and diversified design trend. The digital development gives more development opportunities to logo design.

As an important media for brands to convey information, the logo design shall adapt to the development of the times, grasp the popular pulse of the era, design the representative works that are more suitable for the aesthetic standards and aesthetic needs of the digital information era, so as to promote the continuous innovation and development of logo design.

\section{References}

[1] Liang Wei. Development trend of the logo design from the current logo substitution phenomenon. packaging engineering. 2012(5):133-134

[2] Dawei Li. New trend of logo design in digital era. Popular literature and art.2016,(11): 78-79

[3] Yan Liu. A new perspective for the study of schema teaching method trend of Development about logo graphic. Design.2015,(2): 159-160

[4] Yong lee. The dynamic trend of logo design in digital age. Artistic research. 2011,(2): 158-159

[5] Cheng Lee. On the dynamic development trend of logo design in the digital age. Popular literature and art.2014,(7): 104

[6] Fuzhao Yu. Analysis of dynamic propagation trend of logo design. Popular literature and art.2016,(15): 86 